\title{
Preparation and optimization of calcium fluoride particles for dental applications
}

\author{
Joachim Koeser • Thiago Saads Carvalho • \\ Uwe Pieles · Adrian Lussi
}

Received: 15 January 2014/ Accepted: 16 March 2014/Published online: 30 March 2014

(C) Springer Science+Business Media New York 2014

\begin{abstract}
Fluorides are used in dental care due to their beneficial effect in tooth enamel de-/remineralization cycles. To achieve a desired constant supply of soluble fluorides in the oral cavity, different approaches have been followed. Here we present results on the preparation of $\mathrm{CaF}_{2}$ particles and their characterization with respect to a potential application as enamel associated fluoride releasing reservoirs. $\mathrm{CaF}_{2}$ particles were synthesized by precipitation from soluble $\mathrm{NaF}$ and $\mathrm{CaCl}_{2}$ salt solutions of defined concentrations and their morphology analyzed by scanning electron microscopy. $\mathrm{CaF}_{2}$ particles with defined sizes and shapes could be synthesized by adjusting the concentrations of the precursor salt solutions. Such particles interacted with enamel surfaces when applied at fluoride concentrations correlating to typical dental care products. Fluoride release from the synthesized $\mathrm{CaF}_{2}$ particles was observed to be largely influenced by the concentration of phosphate in the solution. Physiological solutions with phosphate concentration similar to saliva (3.5 mM) reduced the fluoride release from pure $\mathrm{CaF}_{2}$ particles by a factor of 10-20 $\times$ as compared to phosphate free buffer solutions. Fluoride release was even lower in human saliva. The fluoride release could be increased by the addition of phosphate in substoichiometric amounts during $\mathrm{CaF}_{2}$ particle synthesis. The presented results
\end{abstract}

\footnotetext{
J. Koeser $(\bowtie) \cdot$ U. Pieles

Institute for Chemistry and Bioanalytics, University of Applied Sciences and Arts Northwestern Switzerland, Gruendenstrasse 40, 4132 Muttenz, Switzerland

e-mail: joachim.koeser@fhnw.ch

J. Koeser · T. S. Carvalho - A. Lussi

Department of Preventive, Restorative and Pediatric Dentistry, University of Bern, 3010 Bern, Switzerland
}

demonstrate that the morphology and fluoride release characteristics of $\mathrm{CaF}_{2}$ particles can be tuned and provide evidence of the suitability of synthetic $\mathrm{CaF}_{2}$ particles as enamel associated fluoride reservoirs.

\section{Introduction}

Fluoride is well known for its beneficial effect on tooth de-/ remineralization cycles. To keep the levels of fluoride on the enamel surface sufficiently high during the intervals between the application cycles of fluoride containing dentifrice much research has been dedicated to the investigation of continuously releasing fluoride reservoirs. Such reservoirs can be either synthetic fluoride-containing matrices [1-3] or the $\mathrm{CaF}_{2}$-like deposits observed on the enamel surface following the topical application of fluoride [4-6]. The formation of $\mathrm{CaF}_{2}$ particles however is not well understood and little is known about their stability and fluoride release under the conditions of the oral environment and how these parameters might be optimized. While methods have been developed to synthesize defined and fast releasing calcium fluoride particles in vitro $[7,8]$ the factors influencing the formation and morphology of calcium fluoride particles are largely unknown. Recent research on the formation of $\mathrm{MgF}_{2}$ particles by precipitation from soluble magnesium- and fluoride-containing salt solutions has demonstrated that the morphology of such particles with respect to size and shape can be varied by tuning the concentrations of $\mathrm{Mg}^{2+}$ and $\mathrm{F}^{-}$in the starting solutions [9, 10]. Here we present data on defined morphological variations of $\mathrm{CaF}_{2}$ particles prepared by precipitation from soluble $\mathrm{NaF}$ and $\mathrm{CaCl}_{2}$ precursor salt solutions and their optimization as fluoride releasing reservoirs on the enamel surface. 


\section{Materials and methods}

\section{1 $\mathrm{CaF}_{2}$ particle assembly and purification}

$\mathrm{CaF}_{2}$ particles were prepared by rapid mixing of aqueous $\mathrm{CaCl}_{2}$ and $\mathrm{NaF}$ solutions in a 1:1 volume ratio. Precipitates formed within a short time as was evident from the changing turbidity of the samples. The particles were purified after at least $16 \mathrm{~h}$ of assembly by centrifugation at $5,000 \times g$ to $20,000 \times g$, depending on the particle size, washed by resuspension in saturated $\mathrm{CaF}_{2}$ solution, and then followed by a second centrifugation and vacuum drying. For the scanning electron microscopical (SEM) examination $\mathrm{CaF}_{2}$ particles were assessed either directly after the resuspension step or upon resuspension of the dried pellet. A drop of the particle suspension was transfered onto freshly cleaved mica, dried, gold sputter coated and examined in a Zeiss Supra SEM. The $\mathrm{CaF}_{2}$ particles used in this study were produced by a $1: 1$ volumetric mixing of (a) $50 \mathrm{mM} \mathrm{NaF}$ and $250 \mathrm{mM} \mathrm{CaCl}_{2}$ (" $\mathrm{CaF}_{2}-1$ " particles), (b) $50 \mathrm{mM} \mathrm{NaF}$ and $40 \mathrm{mM} \mathrm{CaCl}_{2}$, (c) $10 \mathrm{mM}$ $\mathrm{NaF}$ and $1 \mathrm{M} \mathrm{CaCl}_{2}$ and (d) $8 \mathrm{mM} \mathrm{NaF}$ and $40 \mathrm{mM} \mathrm{CaCl}_{2}$ (" $\mathrm{CaF}_{2}-2$ " particles) (Fig. 1). For experiments investigating the effect of phosphate during $\mathrm{CaF}_{2}$ particle assembly, $1 \mathrm{M}$ phosphate buffer $\mathrm{pH} 7$ was added to the $\mathrm{NaF}$ and $\mathrm{CaCl}_{2}$ solutions to reach final concentrations of either $0.01 \mathrm{mM}$ phosphate, $0.1 \mathrm{mM}$ phosphate or $1 \mathrm{mM}$ phosphate.
2.2 Preparation of tooth enamel specimens and $\mathrm{CaF}_{2}$ particle adhesion assay

All enamel specimens were prepared from caries-free human molar teeth extracted by dental practitioners in Switzerland. Before the extraction, the patients were informed about the use of their teeth for research purposes and consent was obtained. All teeth were stored in $1 \%$ chloramine $\mathrm{T}$ trihydrate solution after the extraction. Human molars were cut using an Isomet ${ }^{\circledR}$ Low Speed Saw (Buehler, Düsseldorf, Germany), separating the crowns from the roots. Subsequently the top of the crown was polished while the other sides were left untreated to produce both a native and a polished tooth enamel surface area. For the enamel polishing the crowns were serially abraded under constant tap water cooling using a Knuth Rotor machine (LabPol 21, Struers, Copenhagen, Denmark) with silicon carbide paper discs of grain size 18, 8 , and $5 \mu \mathrm{m}$, for $60 \mathrm{~s}$ each. As a final step the crowns were polished for $60 \mathrm{~s}$ with $3 \mu \mathrm{m}$ diamond abrasive on Struers polishing cloth under constant cooling (LaboPol-6, DPMol Polishing, DP-Stick HQ, Struers, Copenhagen, Denmark). Between two polishing steps and after the final polishing, all slabs were ultrasonicated for $1 \mathrm{~min}$ in tap water and rinsed. Thus, all prepared specimens had a flat ground enamel area with a $200 \mu \mathrm{m}$ cut off layer. Samples were stored in a mineral solution $\left(1.5 \mathrm{mM} \mathrm{CaCl}_{2}, 1 \mathrm{mM}\right.$ $\mathrm{KH}_{2} \mathrm{PO}_{4}, 50 \mathrm{mM} \mathrm{NaCl}, \mathrm{pH}$ 7.0) and underwent further
Fig. 1 SEM images of calcium fluoride particles prepared by precipitation from $\mathrm{NaF}$ and $\mathrm{CaCl}_{2}$ solutions. Calcium fluoride particles were precipitated by $1: 1$ mixing of soluble $\mathrm{NaF}$ and $\mathrm{CaCl}_{2}$ precursor solutions with molar concentratrations of: a $50 \mathrm{mM}$ $\mathrm{NaF}+250 \mathrm{mM} \mathrm{CaCl}{ }_{2}\left({ }^{\prime} \mathrm{CaF}_{2}-\right.$ 1 ”), b $50 \mathrm{mM} \mathrm{NaF}+40 \mathrm{mM}$ $\mathrm{CaCl}_{2}, \mathbf{c} 10 \mathrm{mM}$

$\mathrm{NaF}+1,000 \mathrm{mM} \mathrm{CaCl}_{2}$ d $8 \mathrm{mM} \mathrm{NaF}+40 \mathrm{mM} \mathrm{CaCl}_{2}$ (" $\mathrm{CaF}_{2}-2$ "). Scale bars are $200 \mathrm{~nm}(\mathbf{a}, \mathbf{b})$ and $2 \mu \mathrm{m}(\mathbf{c}, \mathbf{d})$
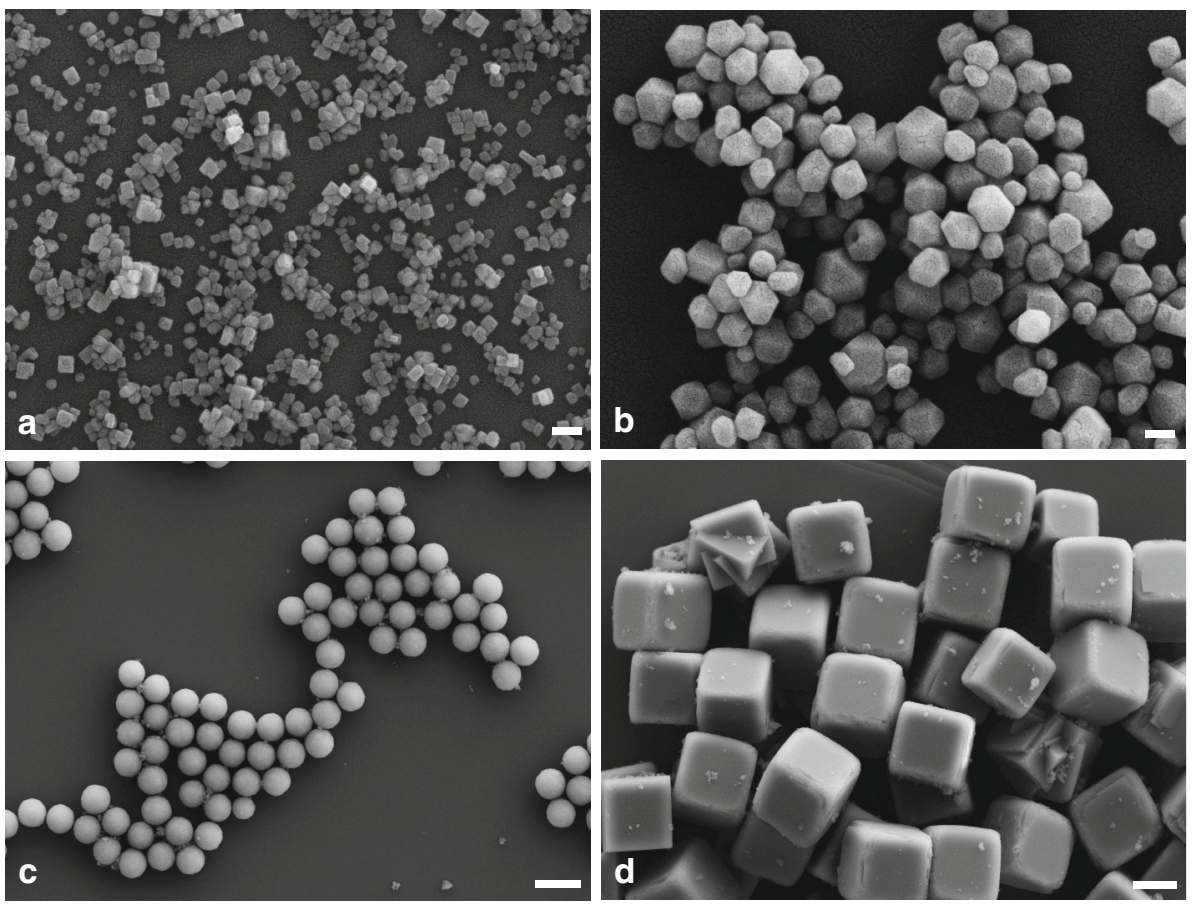
Fig. 2 SEM images of enamel surfaces following incubation with calcium fluoride particles. Enamel surfaces were incubated with calcium fluoride particles in solution, corresponding to $450 \mathrm{ppm}$ fluoride $(\mathbf{a}, \mathbf{c}, \mathbf{f})$ or $1,450 \mathrm{ppm}$ fluoride $(\mathbf{b}, \mathbf{d}, \mathbf{e})$, and for $30 \mathrm{~s}(\mathbf{a}, \mathbf{c}, \mathbf{f})$ or $80 \mathrm{~s}(\mathbf{b}$, d, e). All surface were then washed and examined by SEM. $\mathbf{a}$ and $\mathbf{b}$ " $\mathrm{CaF}_{2}-2$ " particles, $\mathbf{c}-$ f " $\mathrm{CaF}_{2}-1$ " particles. e Shows particle adhesion on polished (left side of the image) and native enamel (right side of the image), $\mathbf{f}$ is a larger magnification of particles adhering to an area of eroded enamel. Scale bars are $10 \mu \mathrm{m}$ $(\mathbf{a}, \mathbf{b}, \mathbf{e})$ and $500 \mathrm{~nm}(\mathbf{c}, \mathbf{d}, \mathbf{f})$
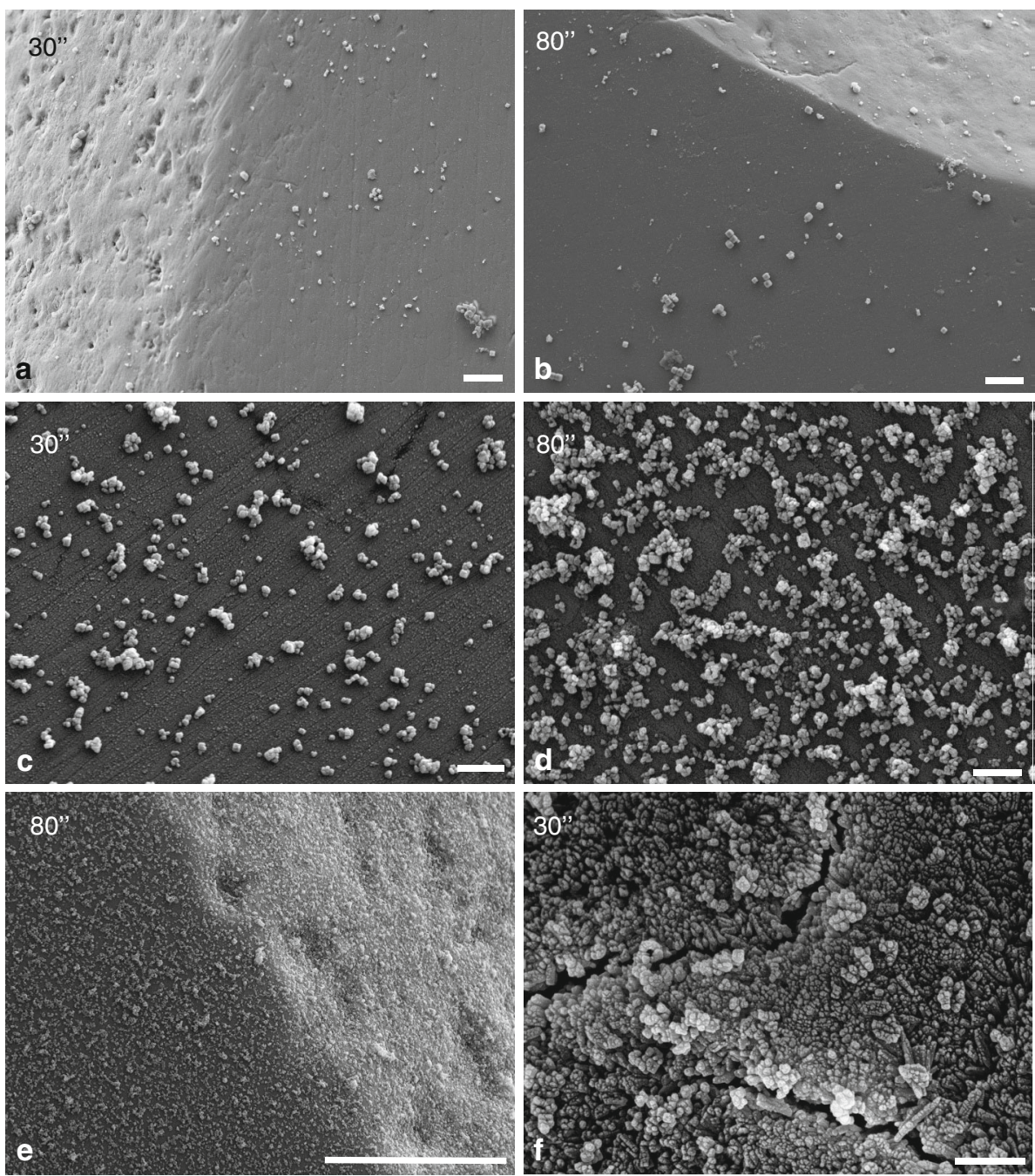

polishing with a $1 \mu \mathrm{m}$ diamond abrasive (60 s, LaboPol-6, DP-Mol Polishing, DP-Stick HQ, Struers, Copenhagen, Denmark) immediately before the experiment.

$\mathrm{CaF}_{2}-1$ and $\mathrm{CaF}_{2}-2$ particles were resuspended in saturated $\mathrm{CaF}_{2}$ solution at amounts corresponding to either $450 \mathrm{ppm}$ or $1,450 \mathrm{ppm}$ fluoride and incubated with the prepared enamel specimens under gentle agitation. Subsequently excess particles were removed by dipping the samples in water several times. The samples they were analyzed by SEM after drying.

\subsection{Image processing and analysis}

For the analysis of the $\mathrm{CaF}_{2}$ particle coating densities on enamel surfaces the Fiji/ImageJ software package was employed [11]. The particle analysis mode was used and the values for the threshold adjustment, particles pixel size and particle circularity were optimized with respect to the brightness, magnification and resolution of the analyzed
SEM images. Images with a large $\mathrm{z}$ range (Fig. 2a, e) were focus stacked using the combineZP software [12].

\subsection{Fluoride release from $\mathrm{CaF}_{2}$ particles}

The fluoride release from different $\mathrm{CaF}_{2}$ particle types was followed over 90 min with a fluoride sensitive electrode (perfectION ${ }^{\mathrm{TM}}$, Mettler Toledo, Greifensee, Switzerland). A total of $10 \mathrm{mg}$ of dried $\mathrm{CaF}_{2}$ particles was mixed with $100 \mathrm{ml}$ of buffer $(20 \mathrm{mM}$ Hepes, $130 \mathrm{mM} \mathrm{KCl}, 1.5 \mathrm{mM}$ $\mathrm{CaCl}_{2}$ either with or without $3.5 \mathrm{mM}$ sodium phosphate, $\mathrm{pH} 7.05$ ) and stirred at $200 \mathrm{rpm}$ with the fluoride sensitive electrode inserted in the solution. Soluble fluoride was measured again at least $24 \mathrm{~h}$ later and the values did not exceed $150 \%$ of the soluble amount detected after $90 \mathrm{~min}$. For the analysis of particle dissolution in saliva, stimulated human saliva was collected in chilled vials, from 30 healthy individuals, $2 \mathrm{~h}$ after their last meal or oral hygiene. The saliva was pooled, centrifuged from $20 \mathrm{~min}$ 
at $4{ }^{\circ} \mathrm{C}(3,000 \mathrm{x} \mathrm{g})$ and separated into $6 \mathrm{ml}$ aliquots. Then, $0.6 \mathrm{mg}$ of dried particles were incubated with $6 \mathrm{ml}$ of pooled stimulated human saliva, stirred at $200 \mathrm{rpm}$ and the fluoride-sensitive electrode inserted in this solution.

\section{Results}

\section{1 $\mathrm{CaF}_{2}$ particle assembly}

$\mathrm{CaF}_{2}$ particles could be produced by precipitation following the mixing of soluble fluoride and calcium salt solutions. For the experiments described here, aqueous $\mathrm{NaF}$ and $\mathrm{CaCl}_{2}$ solutions were used. One to one volumetric mixing of solutions with concentrations of $\mathrm{F}^{-}$ranging from $5 \mathrm{mM}$ to $75 \mathrm{mM}$ and of $\mathrm{Ca}^{2+}$ ranging from $8 \mathrm{mM}$ to $2 \mathrm{M}$ resulted in the precipitation of particles with different morphologies. Some of the different shapes we observed were round, cubic, hexagonal, and irregular formed particles in the size range of approximately $50 \mathrm{~nm}$ to $2 \mu \mathrm{m}$ (see Fig. 1 and [13]). Although the particle shapes could not be predicted in advance a general trend towards larger assemblies at lower parent ion concentrations was observed.

\section{2 $\mathrm{CaF}_{2}$ particle interaction with tooth enamel surfaces}

As a first step in the investigation of the suitability of the prepared $\mathrm{CaF}_{2}$ particles as fluoride reservoirs, their interaction with tooth enamel surfaces was analyzed. Suspensions from two particle types of different size, smaller " $\mathrm{CaF}_{2}-1$ " and larger " $\mathrm{CaF}_{2}-2$ " particles (see Fig. 1), were incubated with human enamel samples at concentrations corresponding to typical dental care product fluoride levels. One surface of each tooth sample had been polished while the rest was left untreated in order to compare the interaction of the particles with 'native' and polished enamel surfaces. Figure 2 shows the results of these experiments. The coating density of the particles on enamel increased with the particle concentration and incubation times. No obvious differences were observed in the interaction of $\mathrm{CaF}_{2}$ particles with polished and unpolished tooth surfaces. The smaller " $\mathrm{CaF}_{2}-1$ " particles covered a larger area as compared to the larger " $\mathrm{CaF}_{2}-2$ " particles. SEM images were analyzed to compare the typical enamel surface coverage with the two different $\mathrm{CaF}_{2}$ particle types. Upon incubation with " $\mathrm{CaF}_{2}-1$ " particles, a coverage of $13 \%$ was reached after $30 \mathrm{~s}$ incubation with $450 \mathrm{ppm}$ fluoride particles and $40 \%$ after $80 \mathrm{~s}$ incubation with $1,450 \mathrm{ppm}$ fluoride particles. The coverage of the enamel surfaces with the larger " $\mathrm{CaF}_{2}-2$ " particles were considerably lower, corresponding to $<1 \%(30 \mathrm{~s}, 450 \mathrm{ppm})$ and $3 \%(80 \mathrm{~s}$, $1,450 \mathrm{ppm})$.

\subsection{Fluoride release from $\mathrm{CaF}_{2}$ particles}

To further investigate the suitability of the synthesized $\mathrm{CaF}_{2}$ particles as fluoride storage reservoirs, their dissolution in physiological buffers was analyzed. These experiments were performed with " $\mathrm{CaF}_{2}-1$ " particles. It turned out that the fluoride release was strongly influenced by the presence of phosphate in the buffer solution which prompted the comparison of the release in buffers without phosphate and with $3.5 \mathrm{mM}$ phosphate, the latter one corresponding approximately to saliva levels [14, 15] (Fig. 3). Under the experimental conditions choosen the levels of soluble fluoride after $90 \mathrm{~min}$ reached $5.33 \pm 0.22 \mathrm{ppm}$ in buffers without phosphate and $0.32 \pm 0.02 \mathrm{ppm}$ in buffers with $3.5 \mathrm{mM}$ phosphate. When incubated with pooled stimulated human saliva the same concentration of particles released approximately $0.1-0.2 \mathrm{ppm}$ fluoride.

\subsection{Enhanced fluoride release from $\mathrm{CaF}_{2}$ particles}

Next, it was investigated if the fluoride release from the $\mathrm{CaF}_{2}$ particles could be influenced by the addition of excipients during their synthesis The literature described a destabilizing effect of phosphate during the formation of $\mathrm{CaF}_{2}$ particles [16]. Thus, a modified protocol for the preparation of $\mathrm{CaF}_{2}$ particles was developed that applied the same $\mathrm{CaCl}_{2}$ and $\mathrm{NaF}$ concentrations as during the preparation of " $\mathrm{CaF}_{2}-1$ " particles, however in the presence of substoichiometric amounts of phosphate. When $\mathrm{CaF}_{2}$ particles were assembled from final concentrations of $25 \mathrm{mM}$ fluoride ions and $125 \mathrm{mM}$ calcium ions, already the addition of as little as $0.01 \mathrm{mM}$ phosphate during

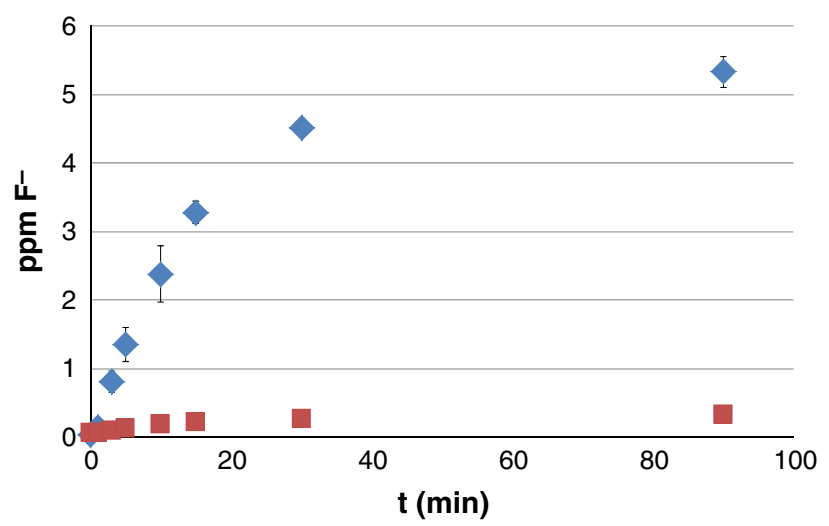

Fig. 3 Time-dependent fluoride release from " $\mathrm{CaF}_{2}-1$ " particles upon incubation in buffers with different phosphate concentrations. Fluoride release (in ppm) from $100 \mathrm{mg} / 1$ " $\mathrm{CaF}_{2}-1$ " particles in buffers with $3.5 \mathrm{mM}$ phosphate (diamonds) or without phosphate (squares) under constant stirring. Error bars for the release experiments in buffers without phosphate are smaller than the size of the symbol 

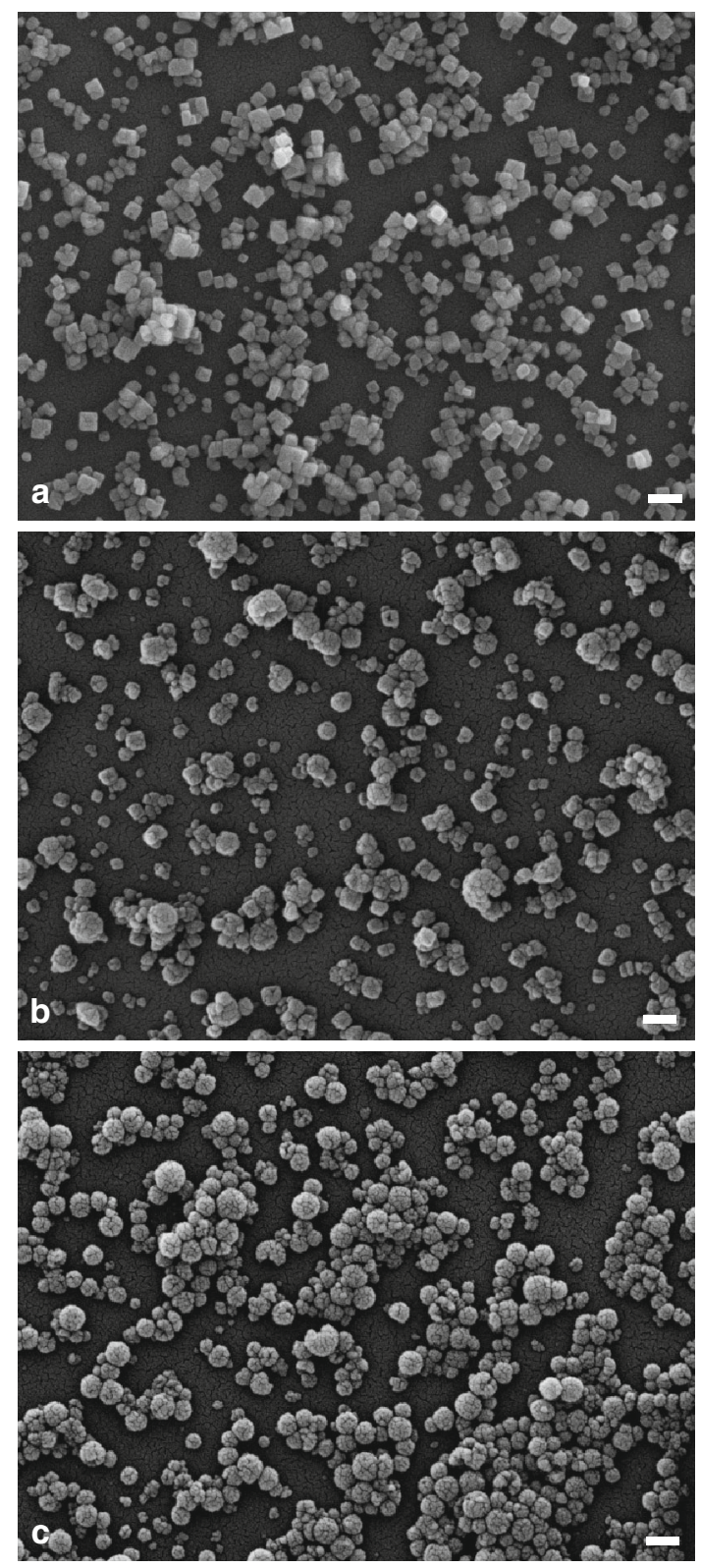

Fig. 4 SEM images of calcium fluoride particles prepared in the presence of different concentrations of phosphate. Calcium fluoride particles were precipitated by $1: 1$ mixing of soluble $\mathrm{NaF}$ and $\mathrm{CaCl}_{2}$ precursor solutions with molar concentratrations of $50 \mathrm{mM} \mathrm{NaF}$ and $250 \mathrm{mM} \mathrm{CaCl} 2$ in the presence of a $0 \mathrm{mM}$ phosphate, b $0.01 \mathrm{mM}$ phosphate and c $1 \mathrm{mM}$ phosphate. Scale bars are $200 \mathrm{~nm}$

assembly had a pronounced effect on the morphology of the resulting particles (Fig. 4). Particles assembled in the presence of phosphate had a more globular appearance and exhibited a rough surface structure. The fluoride release from such synthesized particles in physiological buffers with $3.5 \mathrm{mM}$ phosphate showed marked differences when compared to the release from " $\mathrm{CaF}_{2}-1$ " particles synthesized in the absence of phosphate (Fig. 5). The presence of $0.01 \mathrm{mM}$ phosphate during assembly nearly doubled the solubility of fluoride from the particles. A maximum of
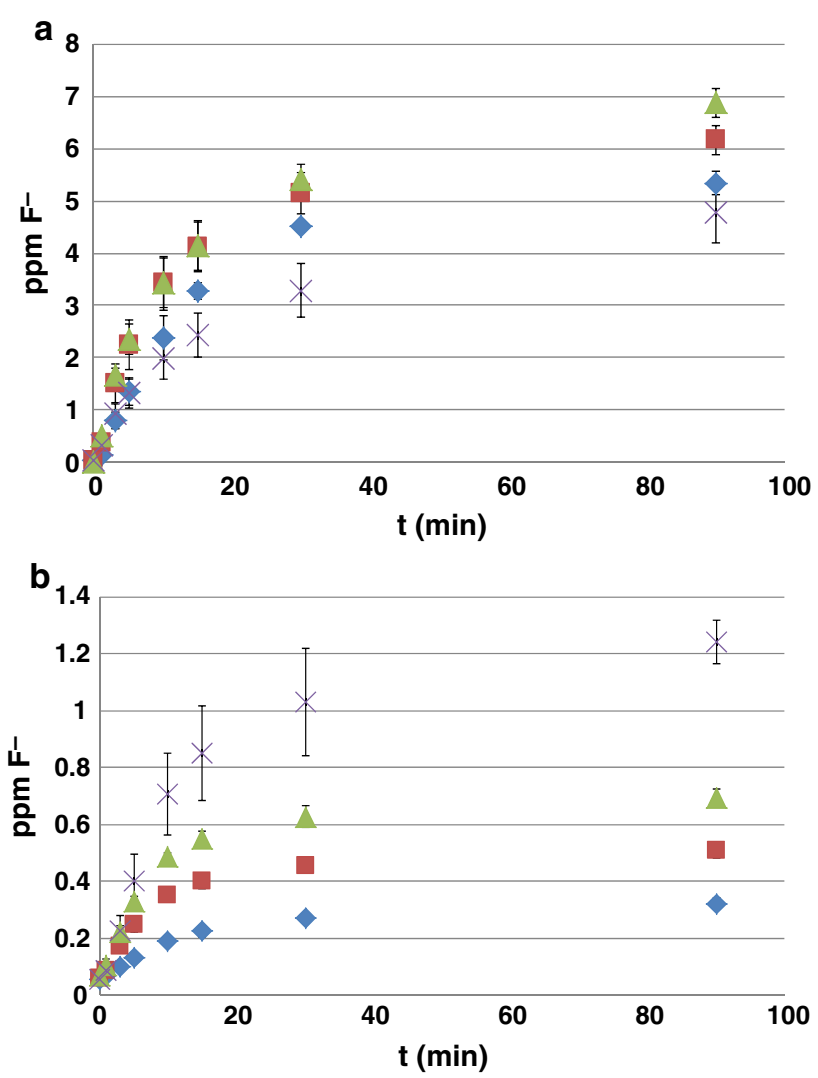

Fig. 5 Influence of phosphate on the fluoride release kinetics from calcium fluoride particles. Fluoride release from calcium fluoride particles synthesized in the presence of $0 \mathrm{mM}$ phosphate (diamonds), $0.01 \mathrm{mM}$ phosphate (squares), $0.1 \mathrm{mM}$ phosphate (triangles) and $1 \mathrm{mM}$ phosphate (crosses) upon resuspension in buffer with $0 \mathrm{mM}$ phosphate (a) or $3.5 \mathrm{mM}$ phosphate (b) at particle concentrations of $100 \mathrm{mg} / \mathrm{l}$. The fluoride release was monitored under constant stirring with a fluoride-sensitive electrode

$1.24 \pm 0.08 \mathrm{ppm}$ soluble fluoride was released from particles assembled in the presence of $1 \mathrm{mM}$ phosphate within $90 \mathrm{~min}$ in $3.5 \mathrm{mM}$ phosphate-containing buffer.

\section{Discussion}

The present study reports results on the preparation of tailor-made $\mathrm{CaF}_{2}$ particles and their suitability as enamel bound fluoride reservoirs for dental care applications.

Generally the formation of $\mathrm{CaF}_{2}$ and $\mathrm{CaF}_{2}$-like material during dental care is limited by the low availability of calcium ions [17]. This problem can be circumvented by the addition of in vitro synthesized $\mathrm{CaF}_{2}$ particles to dental care products.

The results demonstrate that the size and shape of in vitro assembled $\mathrm{CaF}_{2}$ particles can be adjusted in a range between $50 \mathrm{~nm}$ and several micrometers. Globular, cubic, hexagonal or irregular-shaped particles can be generated (see also [13] ). For the experiments presented here, cubic 
rather than round particles were chosen since they were expected to have larger and thus stronger interaction sites with the enamel surface. When such particles were applied to tooth enamel samples in concentrations corresponding to the fluoride content of typical mouth rinses or toothpastes, they interacted with enamel surfaces leading to coverage levels of up to $40 \%$.

Fluoride levels in oral fluids play an important role in the de-/remineralization cycles of tooth enamel. While enamel demineralization is best reduced in the presence of relatively high concentrations of fluoride [18], remineralization benefits from considerably lower concentrations of salivary or plaque fluoride. Epidemiologic studies found a positive correlation between fluoride levels of $0.02 \mathrm{ppm}$ and $0.04 \mathrm{ppm}$ in children and their incidence rate for caries $[19,20]$. Furthermore in vitro studies on remineralization models demonstrated significant enhancement of enamel remineralization in the presence of lower sub ppm levels of fluoride [20]. Generally salivary levels of $0.1 \mathrm{ppm}$ are expected to largely reduce caries even in high risk individuals [21]. Under the experimental condition chosen in the present report, the fluoride release characteristics of the calcium fluoride particles resulted in soluble fluoride levels well above $0.3 \mathrm{ppm}$ if particles were synthesized in the absence of soluble phosphate and above $1 \mathrm{ppm}$ if prepared in the presence of $1 \mathrm{mM}$ phosphate.

The results presented here represent data from in vitro studies and the conditions on the enamel surface in the oral cavity most likely will influence the fluoride release in vivo. An in situ study conducted by Tenuta et al. [22] investigated plaque fluid fluoride levels in Streptococcus mutans test plaques in contact with in vitro generated $\mathrm{CaF}_{2}$ deposits in the oral cavity and their correlation with surface microhardness changes upon a cariogenic challenge. Their results showed a clear positive correlation between the presence of $\mathrm{CaF}_{2}$ deposits, fluoride levels in the plaque fluid and reduced changes in surface microhardness after a cariogenic challenge. The protective effect was strongest with freshly deposited $\mathrm{CaF}_{2}$, however even after $48 \mathrm{~h}$ of aging in artificial saliva, surface microhardness losses were reduced by more than a factor of two as compared to $\mathrm{CaF}_{2}$ free samples. The amounts of $\mathrm{CaF}_{2}$ deposited in these experiments were approximately $20 \mu \mathrm{g}$ fluoride per $\mathrm{cm}^{2}$, which is comparable to the amounts deposited when the present " $\mathrm{CaF}_{2}-1$ " particles interacted with enamel surfaces. More specifically, assuming an average particle size of $100 \mathrm{~nm}$, the amount deposited can be calculated to be in the range of $12 \mu \mathrm{g}$ fluoride per $\mathrm{cm}^{2}$ for the $40 \%$ coverage rate observed after $80 \mathrm{~s}$ of incubation (Fig. 2). Future work will have to focus on the long-term fluoride release from the $\mathrm{CaF}_{2}$ particles presented here under the conditions of the oral cavity. The literature describes the formation of a $\mathrm{pH}$-dependent protective phosphate layer on the surface of
$\mathrm{CaF}_{2}$ particles in physiological solutions containing soluble phosphate and which influences the release of fluoride from such particles over time and during cariogenic challenges [23-25]. The formation of such a phosphate layer can explain the results of the present study, where the levels of soluble fluoride were lower in buffers containing phosphate in relation to phosphate-free buffers. Furthermore, the formation of a layer could also explain the reduced fluoride release observed after $48 \mathrm{~h}$ of aging of the $\mathrm{CaF}_{2}$ deposits in the publication by Tenuta et al. [22]. However, there is still a sufficient amount of fluoride released to have a beneficial effect during a cariogenic challenge, and several authors report an increased fluoride release from phosphate coated $\mathrm{CaF}_{2}$ under the acidic conditions of a cariogenic challenge $[17,26,27]$. The latter scenario provides support for the potential of synthesized $\mathrm{CaF}_{2}$ particles serving as caries reducing enamel associated fluoride reservoirs.

The formation of the phosphate layer has been regarded as the major mechanism influencing $\mathrm{CaF}_{2}$ solubility in saliva [24]. When investigating the solubility of $\mathrm{CaF}_{2}$ in water, $2 \mathrm{mmol} / \mathrm{l}$ phosphate solution or saliva, Saxegaard et al. [24] observed that the rate of dissolution of $\mathrm{CaF}_{2}$ was comparably lower in both saliva and in the phosphate solution. In our experimental model, the solubility of $\mathrm{CaF}_{2}$ did decrease in the phosphate solution $(3.5 \mathrm{mM})$, possibly due to the phosphate layer, but, strikingly, the reduction in $\mathrm{CaF}_{2}$ solubility was even more pronounced in the presence of saliva. In that case, the presence of other compounds in saliva, such as proteins from the salivary pellicle, also affect the dissolution of $\mathrm{CaF}_{2}$ [28]. When the tooth is exposed to saliva, pellicle precursor proteins, such as statherin, almost immediately interact with the tooth surface, thus triggering the initial stages of a perm-selective pellicle formation [29]. The salivary pellicle is then able to modify the transport of calcium ions to and from the tooth surface, which also explains the lower solubility of $\mathrm{CaF}_{2}$ in saliva.

In vivo, the precipitation of $\mathrm{CaF}_{2}$ particles onto the tooth surface usually occurs in the presence of the salivary pellicle. These $\mathrm{CaF}_{2}$ particles are often described as having a globular spherical shape with a nodular surface [4]. Remarkably, our results showed that such structures were often found when $\mathrm{CaF}_{2}$ particles were formed in the presence of phosphate. Furthermore, the presence of phosphate during particle formation also influenced the $\mathrm{CaF}_{2}$ solubility, where the addition of substoichiometric amounts of phosphate during $\mathrm{CaF}_{2}$ particle synthesis was able to considerably increase fluoride solubility. Consequently, the presence of saliva in vivo can serve as a source for phosphate during particle assembly, thus leading to globular spherical shaped, albeit more soluble, $\mathrm{CaF}_{2}$ particles. On the other hand, the presence of a salivary film may lead to greater amounts of $\mathrm{CaF}_{2}$ formed on the tooth surface [30], 
as well as to the formation of the phosphate layer and salivary pellicle over the particles, which is related to lower solubility. Nevertheless, more studies are still necessary to further elucidate the effect of the interaction of phosphate during and after particle assembly, as well as the effect of salivary proteins on the solubility of the $\mathrm{CaF}_{2}$ particles in vivo.

Future experiments could combine the particle coverage rates of enamel described here with typical saliva dynamics and ion diffusion rates in the dental plaque to give information on the in vivo fluoride reservoir properties of the present $\mathrm{CaF}_{2}$ particles described here. Of special interest in this respect will be the examination of calcium fluoride particles in caries models working with bacterial biofilms.

\section{Conclusion}

The main finding of the presented study is that $\mathrm{CaF}_{2}$ particle assembly is influenced by the concentrations of $\mathrm{Ca}^{2+}$ and $\mathrm{F}^{-}$ions in the parent salt solutions and the presence of modifying compounds. This offers the possibility to tune the morphology and fluoride release kinetics of such particles to suit the specific requirements of different topical applications of fluoride containing dental care products.

Acknowledgments The Swiss Nanoscience Institute and the University of Bern (Department of Preventive, Restorative and Pediatric Dentistry) financed the research presented here. The support from the members of the research groups of Prof. U. Pieles and Prof. A. Lussi is gratefully acknowledged.

\section{References}

1. Pessan JP, Al-Ibrahim NS, Buzalaf MA, Toumba KJ. Slowrelease fluoride devices: a literature review. J Appl Oral Sci. 2008;16:238-46.

2. Wiegand A, Buchalla W, Attin T. Review on fluoride-releasing restorative materials-fluoride release and uptake characteristics, antibacterial activity and influence on caries formation. Dent Mater. 2007;23:343-62.

3. Gambhir R, Kapoor D, Singh G, Singh J, Kakar H. Intraoral fluoride-releasing devices: a literature review. World J Dent. 2012:350-354.

4. Petzold M. The influence of different fluoride compounds and treatment conditions on dental enamel: a descriptive in vitro study of the $\mathrm{CaF} 2$ precipitation and microstructure. Caries Res. 2001;35:45-51.

5. Nelson DG, Jongebloed WL, Arends J. Morphology of enamel surfaces treated with topical fluoride agents: SEM considerations. J Dent Res. 1983;62:1201-8.

6. Duschner H, Götz H, Ogaard B. Fluoride-induced precipitates on enamel surface and subsurface areas visualised by electron microscopy and confocal laser scanning microscopy. Eur J Oral Sci. 1997; 105:466-72.

7. Sun L, Chow LC. Preparation and properties of nano-sized calcium fluoride for dental applications. Dent Mater. 2008;24:111-6.
8. Sun L, Chow LC, Bonevich JE, Wang T, Mitchell JW. A new approach to prepare well-dispersed $\mathrm{CaF}(2)$ nanoparticles by spray drying technique. J Biomed Mater Res B Appl Biomater. 2011;98B:223-9.

9. Sevonkaev I, Matijević E. Formation of magnesium fluoride particles of different morphologies. Langmuir. 2009;25:10534-9.

10. Nandiyanto $A B$, Iskandar $F$, Ogi $T$, Okuyama $K$. Nanometer to submicrometer magnesium fluoride particles with controllable morphology. Langmuir. 2010;26:12260-6.

11. Schindelin J, Arganda-Carreras I, Frise E, Kaynig V, Longair M, Pietzsch T, et al. Fiji: an open-source platform for biologicalimage analysis. Nat Methods. 2012;9:676-82.

12. Hadley A. CombineZP image stacking software. http://www. hadleyweb.pwp.blueyonder.co.uk/2008. Accessed date 13, 2012.

13. Koeser J, Pieles U. Towards optimized fluoride particles for dental care applications. Eur Cells Mater. 2012;23(Suppl. 2):19.

14. Bardow A, Lagerlöf F, Nauntofte B, Tenuovo J. The role of saliva. In: Fejerskov O, Kidd E, editors. Dental caries: the disease and its clinical management. Oxford: Blackwell Munksgaard Ltd.; 2008. p. 190-2007.

15. Anderson P, Hector M, Rampersad MC. PiraswsigocaaIJPD, 11:266-273. Critical $\mathrm{pH}$ in resting and stimulated whole saliva in groups of children and adults. Int J Paediatr Dent. 2001:266-273.

16. Christoffersen J, Christoffersen M, Kibalczyc W, Perdok W. Kinetics of dissolution and growth of calcium-fluoride and effects of phosphate. Acta Odontol Scand. 1988;46:325-36.

17. Vogel GL. Oral fluoride reservoirs and the prevention of dental caries. Monogr Oral Sci. 2011;22:146-57.

18. Hughes J, West N, Addy M. The protective effect of fluoride treatments against enamel erosion in vitro. J Oral Rehabil. 2004;31:357-63.

19. Leverett D, Featherstone J, Proskin H, Adair S, Eisenberg A, Mundorffshrestha $\mathrm{S}$, et al. Caries risk assessment by a crosssectional drscrimination model. J Dent Res. 1993;72:529-37.

20. Gibbs C, Atherton S, Huntington E, Lynch R, Duckworth R. Effect of low-levels of fluoride on calcium-uptake by demineralized human enamel. Arch Oral Biol. 1995;40:879-81.

21. Featherstone JD. Delivery challenges for fluoride, chlorhexidine and xylitol. BMC Oral Health. England. 2006. p S8.

22. Tenuta LM, Cerezetti RV, Del Bel Cury AA, Tabchoury CP, Cury JA. Fluoride release from CaF2 and enamel demineralization. J Dent Res. 2008;87:1032-6.

23. Rolla G, Ekstrand J. Fluoride in oral fluids and dental plaque. In: Fejerskov O, Ekstrand J, Burt BA, editors. Fluoride in dentistry, 2nd edn. 1996. p. 215-229.

24. Saxegaard E, Lagerlof F, Rolla G. Dissolution of calcium-fluoride in human saliva. Acta Odontol Scand. 1988;46:355-9.

25. Chander S, Chiao C, Fuerstenau D. Transformation of calciumfluoride for caries prevention. J Dent Res. 1982;61:403-7.

26. Vogel G, Zhang Z, Chow L, Carey C, Schumacher G, Banting D. Effect of in vitro acidification on plaque fluid composition with and without a $\mathrm{NaF}$ or a controlled-release fluoride rinse. J Dent Res. 2000;79:983-90.

27. Rolla GE, J. Fluoride in oral fluids and dental plaque. In: Fejerskov O, Ekstrand J, Burt BA, editors. Fluoride in dentistry, 2nd edn. 1996. p. 215-29.

28. Ganss C, Schlueter N, Klimek J. Retention of KOH-soluble fluoride on enamel and dentine under erosive conditions-a comparison of in vitro and in situ results. Arch Oral Biol. 2007;52:9-14.

29. Siqueira W, Custodio W, McDonald E. New insights into the composition and functions of the acquired enamel pellicle. J Dent Res. 2012;91:1110-8.

30. Larsen M, Richards A. The influence of saliva on the formation of calcium fluoride-like material on human dental enamel. Caries Res. 2001;35:57-60. 